\title{
Pulmonary outflow stenosis due to saphenous vein graft aneurysm: a rare case report and review of literature
}

\author{
Eyad Jaara $^{1 *}$, Mohammed Al-Natour ${ }^{2}$ and Haitham Elsamaloty ${ }^{3}$ \\ ${ }^{1} \mathrm{MS} 3$, The University of Toledo, USA \\ ${ }^{2}$ Senior Radiology Resident, The University of Toledo, USA \\ ${ }^{3}$ Professor \& Interim Chairman, Department of Radiology, The University of Toledo, USA
}

\begin{abstract}
Saphenous Vein Graft Aneurysms (SVGA) have been reported as a rare complication of post-coronary artery bypass grafting (CABG) in the literature. Complications typically arise 10 to 20 years post CABG. Acquired pulmonary artery trunk stenosis in adults is another rare entity that may be caused by a SVGA, among other etiologies. The finding was incidentally discovered on CTA performed to rule out pulmonary embolism on a post hip-fracture repair patient who was experiencing shortness of breath. The diagnosis was confirmed by CTA coronary study with multiple 3-D rotational volumetric-surface shaded images. Therefore cross sectional imaging can give early and accurate measurements of the size of an aneurysm and its relationship to adjacent structures, emphasizing its added value in early diagnosis of SVGA.
\end{abstract}

\section{Introduction}

Saphenous Vein Graft Aneurysms (SVGA) have been reported as a rare complication of post coronary artery bypass grafting (CABG) in the literature. Complications typically arise 10 to 20 years post CABG, with common etiologies being vessel wall ischemia, atherosclerotic degeneration, among other causes [1]. In 1975, Riahi described the first reports of such a phenomenon [2] and distinguished that the incidence of a minor dilation of saphenous vein grafts varies up to $14 \%$, whereas the significant aneurysm is unusual at merely $0.07 \%[3,4]$. Since that time, SVGA has become increasingly encountered as the advances in medicine have prolonged the life expectancy of bypass graft recipients. SVGA most often presents as chest pain, but can possibly present with symptoms of abdominal pain as well [5]. Optimal methods for diagnosing SVGA include the use of computed tomography (CT) and or magnetic resonance imaging (MRI), as both are more effective than coronary angiography in terms of estimating the true size of the aneurysm [1]. Treatment algorithms include exclusion of the aneurysm with a stent, or open surgery if the former is not a viable option [6].

Acquired pulmonary artery trunk stenosis in adults is another rare entity. Reviews have suggested multiple intrinsic and extrinsic causes, including: bronchial carcinoma, mediastinal neoplasms and fibrosis, ascending aortic aneurysms, Takayasu arteritis, intraluminal filling defects due to pulmonary thromboembolism, and pulmonary artery sarcomas [7].

We present the rare case of a large aortocoronary bypass saphenous vein graft aneurysm causing significant extrinsic compression on the main pulmonary artery trunk outflow. The finding was incidentally discovered on CTA performed to rule out a pulmonary embolism on a post hip-fracture repair patient who was experiencing shortness of breath. The diagnosis was confirmed by a CTA coronary study with multiple 3-D rotational volumetric-surface shaded images and angiography. In a review of the literature, we found only six cases that describe SVGA causing pulmonary trunk stenosis.

\section{Case report}

A 79-year-old Caucasian male presented to our service for evaluation of progressively worsening shortness of breath after undergoing open reduction and internal fixation of an intertrochanteric fracture of the right hip. On the third post-operative day, the patient developed acute progressive shortness of breath with cough. His past medical history included hypertension, hyperlipidemia, chronic kidney disease stage III, recent diagnosis of prostate cancer undergoing treatment, and coronary artery disease. Seventeen years earlier he had undergone aortocoronary grafting using three saphenous vein grafts to the obtuse marginal artery $(\mathrm{OM})$, posterior descending artery (PDA) and diagonal artery and a fourth left internal mammary artery (LIMA) graft to the left anterior descending artery (LAD). The patient was non-compliant with his medication after the operation. The patient has a nine-pack year history but had quit 54 years ago.

On physical examination, the patient's blood pressure was 159/76 $\mathrm{mm} \mathrm{Hg}$, his pulse was normal and regular, his respiratory rate was eighteen breaths per minute, and he had a normal temperature. Oxygen saturation measured $93 \%$ on two liters nasal cannula. The patient had a soft systolic ejection murmur at the right upper sternal border, and his lung sounds were clear on auscultation except for scant crackles at the bases.

A work-up was initiated to rule out myocardial infarct, pulmonary embolism, and CHF. Initial cardiac markers were normal, and the electrocardiogram demonstrated sinus rhythm with an old inferior

Correspondence to: Eyad Jaara, MS3 The University of Toledo, 5328 Palisades Drive Sylvania, Ohio 43560, USA,Tel: 4192793573; E-mail: ejaara@rockets. utoledo.edu

Received: August 15, 2017; Accepted: September 07, 2017; Published: September 09, 2017 
infarct pattern and nonspecific ST changes. Echocardiogram revealed inferior wall akinesis, an ejection fraction estimated at 35-40\%, and an elevated right ventricular systolic pressure measuring $40-50 \mathrm{mmHg}$. A chest radiograph demonstrated a $5.5 \mathrm{~cm} \mathrm{x} 6 \mathrm{~cm}$ anterior left hilar mass (Figure 1). A chest CT scan with contrast was concerning for a SVGA (Figure 2). A large aneurysm of the diagonal artery bypass vein graft which measured $5.5 \mathrm{~cm} \times 5.9 \mathrm{~cm}$ was confirmed by coronary CTA study (Figure 3) with multiple 3-D rotational volumetric surface shaded image (Figure 4). The aneurysm was located anterolateral to the aortopulmonic window region and was compressing the adjacent pulmonary outflow with a transverse dimension of $2 \mathrm{~cm}$. It was predominantly filled with thrombus and about $30 \%$ of its lumen opacified with contrast medium (Figure 3). Further workup included a pulmonary function test, which revealed a mild degree of restrictive and obstructive ventilatory impairment, along with a decrease in diffusion capacity and a moderate impairment of gas transfer capacity. Pulmonary embolism studies were consequently ordered.

The patient underwent a cardiac catheterization demonstrating a pseudoaneurysm of the saphenous vein graft bypassing the diagonal artery. The study also found minimal blood flow distal to the aneurysm. Given the patient's comorbidities, he was not a candidate for repeat CABG. A percutaneous coil-embolization was successfully performed to occlude flow to the aneurysm due to its high risk of rupture. A Cook $2 \mathrm{~mm} \times 3 \mathrm{~cm}$ embolization coil was placed into the distal portions of the proximal limb of the saphenous vein graft, completely stopping flow in an anterograde fashion. The patient did have a subsequent mild non-ST elevation MI, but was stable hemodynamically and clinically. The patient did well after the procedure and was discharged after a few days. In a follow up visit three months later, he did not develop any

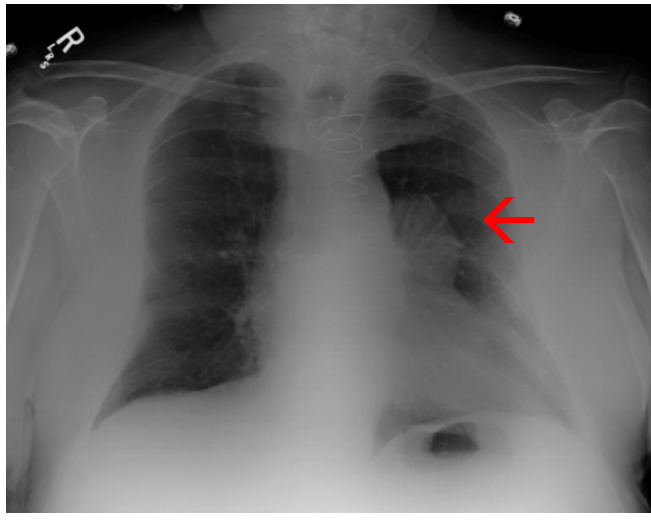

a

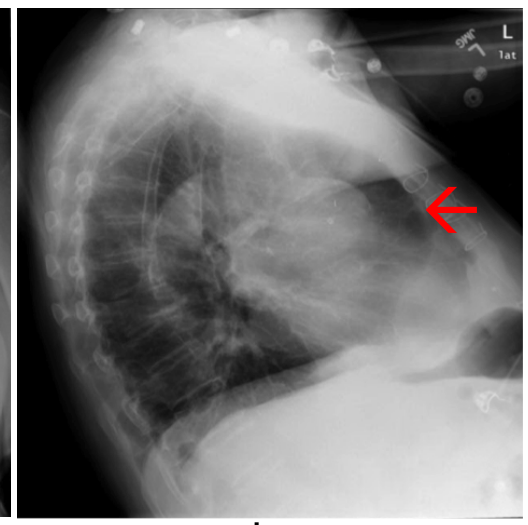

b

Figure 1. AP and lateral chest X-ray demonstrating a large left perihilar mass anteriorly (red arrow)

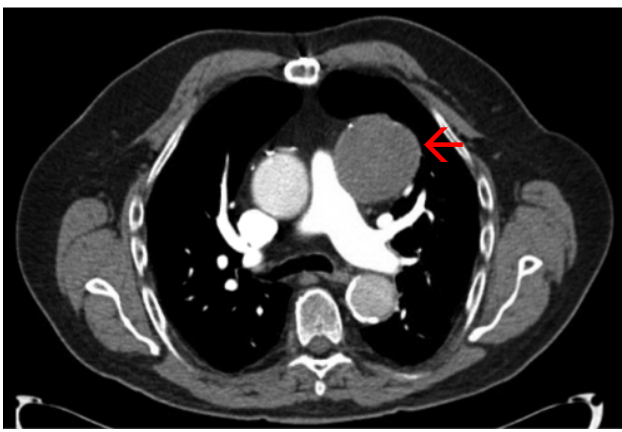

a

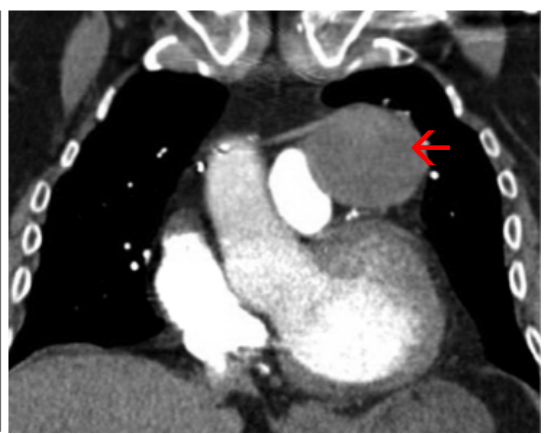

b

Figure 2. Chest computed tomography with contrast, pulmonary embolism protocol, showing anterior mediastinal mass (red arrow) compressing and narrowing the pulmonary outflow tract

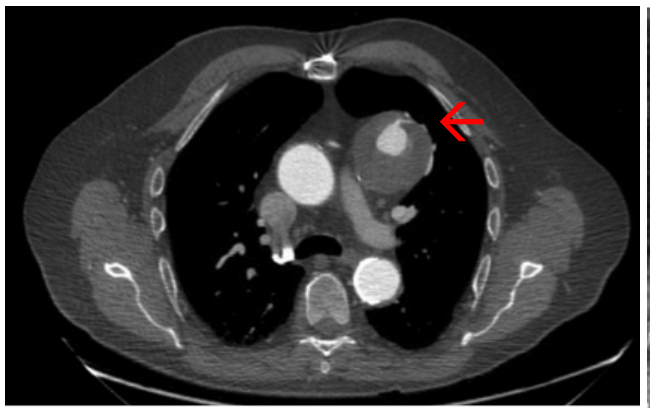

a

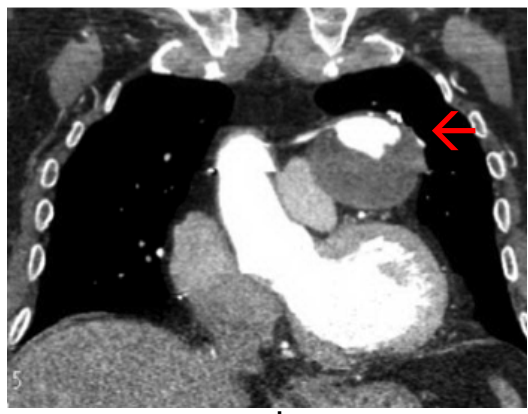

b

Figure 3. Gated coronary computed tomography angiogram showing saphenous vein graft aneurysm (red arrow) with contrast-filled small $2 \mathrm{~cm}$ true lumen surrounded by large thrombus. 


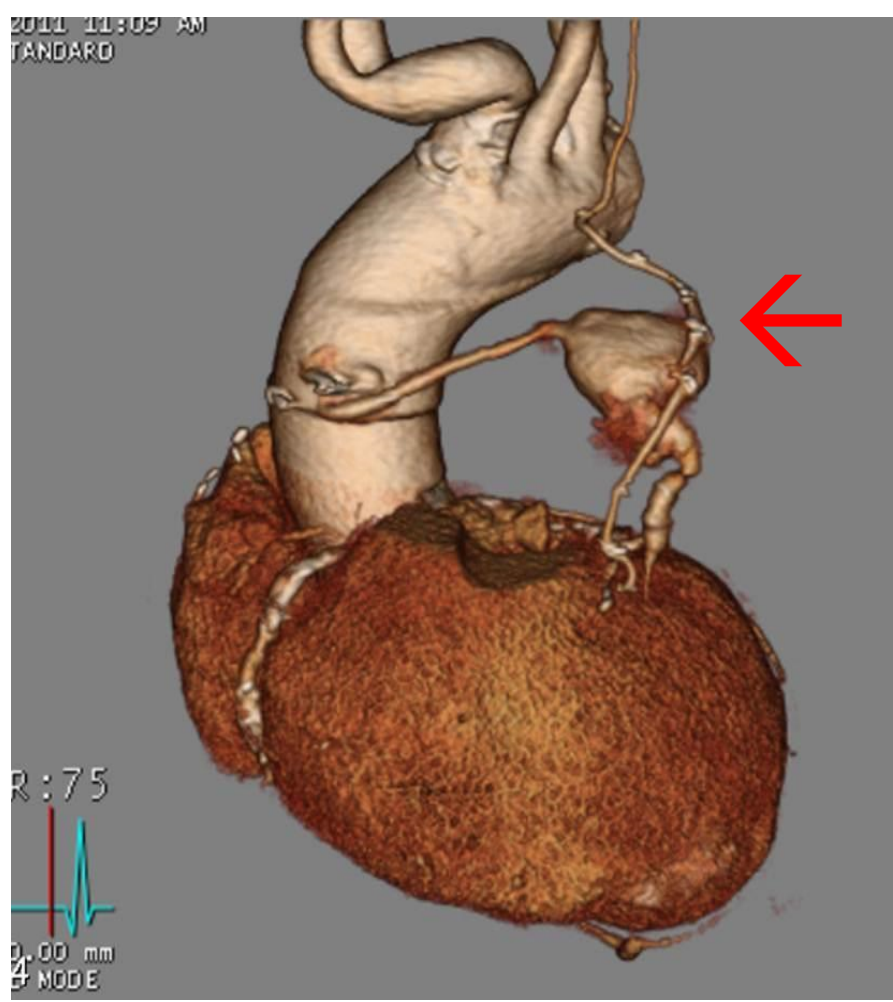

Figure 4. 3D Volumetric surface-shaded image showing patent LIMA graft to distal LAD and saphenous vein graft to diagonal artery with aneurysm (red arrow) in the middle segment

further symptoms, and a computed tomographic scan demonstrated no change in the aneurysm dimensions.

\section{Discussion}

Coronary artery bypass graft surgery with the saphenous vein has been considered a surgical standard for the treatment of coronary artery disease since 1967. Saphenous vein graft aneurysms, a rare complication of CABG, have also been reported. An aneurysm is defined as a permanent localized dilatation that contains the entire vascular wall, resulting in at least a $50 \%$ increase in arterial or venous diameter compared to the normal expected diameter ${ }^{7}$. Two reported types of SVGA are aneurysms and pseudoaneurysms. True aneurysms are primarily due to accelerated atherosclerosis and involve the entire vessel wall [8]. A pseudoaneurysm, or false aneurysm, on the other hand, is caused by a breach in a vessel wall, causing a blood-leak that is contained by the adventitia or surrounding perivascular soft tissue. Furthermore, it lacks an intimal layer, as it arises from disruption between the media and adventitia [9]. Pseudoaneurysms seen in grafts mainly occur at suture lines, and are most often due to breakage of a suture, dehiscence of a suture line or by infections that are either direct or that are hematogenous seed to the anastomotic sites at the proximal or distal ends of the vein graft [10-12]. Due to such pathophysiology, pseudoaneuryms are commonly seen as an acute finding whereas true aneuryms caused by atherosclerosis, are more subclinical and chronic in nature. It is also important to note that true aneurysms are more common than pseudoaneurysms by a ratio of $6: 1$ [13]. Because the differentiation between a pseudoaneurysm and true aneurysm is pathologically determined and not always delineated, the term "aneurysm" will be used in the remainder of this report.

A large SVGA is described as being $4 \mathrm{~cm}$ or more in diameter [14]. They are mostly asymptomatic and discovered incidentally on routine chest radiographic examinations [14]. Patients with graft aneurysms have also been reported to present with myocardial ischemia; aneurysms are often thrombus-filled and may lead to occlusion and periodic emboli. Multiple life-threatening complications in SVGA have been reported including myocardial infarction [15], rupture [16], fistula formation to different heart chambers and adjacent major vessels, and even death when they go undetected [14]. Other reported complications from the compressive effects of SVGA include acute superior vena cava obstruction [17] cardiac tamponade [7] and pulmonary artery stenosis $[18,19]$.

In our review of the literature, we found only six reports of SVGA causing pulmonary trunk compression. The first case was discovered incidentally by routine chest radiography and showed a large mass adjacent to the left heart border. A contrast-enhanced multi-detector row spiral CT scan of the chest confirmed the diagnosis of 2 large true SVGAs. The first aneurysm was found in a bypass graft to the circumflex coronary artery measuring $8 \times 9 \times 8 \mathrm{~cm}$ with considerable compression on pulmonary trunk. The second one was an aneurysm of the bypass grafts to the right coronary artery. The patient died suddenly from an infra-renal abdominal aortic aneurysm rupture before any management of the SVGA could be done [19].

The second case was a large aneurysm seen in a SVG feeding a diagonal artery. The aneurysm compressed the patient's pulmonary trunk, causing angina on exertion followed by right-sided heart failure three months later. CT scan confirmed a $7 \mathrm{~cm}$ aneurysm and surgical intervention including thrombus removal, re-grafting and pulmonary artery repair was undergone with an uneventful recovery [20].

In the next report, a patient presents with a non-ST elevation myocardial infarction and exertional dyspnea nineteen years after a CABG surgery. A CT scan and reconstructed CTA coronary showed an $8 \mathrm{~cm}$ in diameter SVG aneurysm arising from the OM graft and compressing the main pulmonary artery. Surgical intervention including thrombus removal and re-grafting was successfully done [21].

The patient in the fourth reported case presented with non-resolving resting chest pain and chest radiograph revealed a mass in the middle mediastinum. Coronary angiography revealed total occlusion of the SVG to the LAD artery with an aneurysmal sac. Intraoperative contrast transesophageal echocardiography identified a large SVG aneurysm with diameters of $5.5 \times 6.0 \mathrm{~cm}$ compressing the main pulmonary artery. The patient underwent surgical resection of the aneurysm followed by replacement of the bypass graft [22].

A fifth patient was admitted with a lower respiratory tract infection when a chest $\mathrm{x}$ ray incidentally suggested a large anterior mediastinal mass. Subsequent CT and cardiovascular MRI demonstrated a $10.6 \times 9$ $\mathrm{cm}$ SVGA of the graft to the OM artery causing significant compression of the aorta and pulmonary artery. The patient was conservatively managed [23].

The last reported case is of a patient admitted for worsening dyspnea and atypical chest pain. Chest X-ray revealed a left anterior mediastinal mass and radiological investigation with enhanced spiral chest CT scan revealed a $7-\mathrm{cm}$ SVGA compressing the left atrium as well as the left pulmonary artery. This was subsequently confirmed by aortography demonstrating an aneurysm of the bypass graft to the circumflex artery. Surgical intervention including thrombus removal and re-grafting was successfully done [24].

In 2005, Almanaseer and his colleague reviewed 108 cases of SVGA and concluded that the diagnosis should be suspected in patients who 
have undergone CABG and present with a mediastinal mass on chest $\mathrm{X}$-ray. They further suggested that SVGAs radiographically visualized in the right sternal border mainly represent SVGAs bypassing the right coronary artery and that SVGAs seen in the left upper sternal border seem to typically bypass the left anterior descending artery. Ones seen in the left lower sternal border were found to be bypassing the LAD and left circumflex artery or both [13].

In the cases of pulmonary trunk stenosis reviewed, including our own, the SVGAs causing pulmonary stenosis were predominantly visualized in the left upper sternal border on Chest X-ray. However, there was greater variance in the artery being bypassed. 2 cases SVGAs to the circumflex artery, 2 were of the OM artery, 2 of the diagonal artery including our case, and 1 was of the LAD artery.

In our case a large $5.5 \mathrm{~cm} \times 5.9 \mathrm{~cm}$ aneurysm was seen in the SVG bypassing the diagonal artery. The aneurysm caused significant pulmonary artery trunk outflow stenosis and was significant enough to mimic the symptomatology of pulmonary thromboembolism. The absolute size of a SVGA can correlate with symptomatology and is useful in deciding a management plan. Coronary angiography can help in the diagnosis of SVGA; however it seems to underestimate size due to the fact that most of SVGAs are filled with thrombus as was seen in our case. On the other hand, cross sectional imaging seems to provide precise estimates of the size of an aneurysm, its location, and its relation to surrounding structures making it more useful $[9,21]$.

The patient in our case presented with shortness of breath upon exertion preoperatively. His shortness of breath progressively worsened postoperatively and this was thought to be due to further compromise of lung functions from general anesthesia and atelectasis.

Treatment options of SVG aneurysm include medical therapy with surveillance, surgical therapy, and percutaneous intervention [25]. Larger SVG aneurysms appear to benefit mainly from aggressive intervention, due to increased rates of rupture and cardiac death [10]. A conservative or interventional approach to treatment of a SVGA should be based upon the size and rate of growth in association with the patient's health status [4].

While the optimal timing of surgery is unknown, in cases of symptomatic aneurysms, fistula formation, suspected mycotic aneurysm, urgent surgical intervention is strongly recommended. The traditional surgical approach has been ligation of the aneurysmcontaining SVG and placement of a new bypass graft however other approaches have been utilized.

While percutaneous procedures were typically reserved as SVGA treatment in poor surgical candidates, they have quickly advanced to become an alternative to surgical intervention. The most common percutaneous approach is coil embolization of the aneurysm. The technique carries the risk of occluding flow to the bypassed arterial system. Coil embolization of the diagonal artery bypass graft was employed in our case due to the patient's co-morbidities as well as the minimal blood flow distally with successful obliteration of the aneurysm.

\section{Conclusion}

The diagnosis of SVGAs remain a challenge for physicians. Such lesions have a peculiar appearance on chest radiographic examinations and should be highly considered when a chest $\mathrm{x}$-ray in a patient with prior heart surgery reveals a mediastinal, hilar, or paracardiac mass. Due to advances in medicine that have prolonged the life expectancy of bypass graft recipients, a higher prevalence of SVGA may be in the horizon.

SVGAs have a distinct appearance on angiography with sizes that are usually underestimated due to intraluminal thrombosis. Cross sectional imaging on the other hand can give accurate estimates of the size of an aneurysm and can further aid in localization to adjacent structures emphasizing its added value in early diagnosis of SVGA. We hope that these considerations will enable a more timely and focused diagnosis of such cases. With conservative or surgical management of the symptomatic SVGA, more dire complications such as rupture can be prevented.

\section{References}

1. Zientara A, Dzemali O, Odavic D, Genoni M (2014) Saphenous vein graft aneurysms: a growing problem? Presentation of two cases and surgical management. Thorac Cardiovasc Surg Rep 3: 23-26. [Crossref]

2. Riahi M, Vasu CM, Tomatis LA, Schlosser RJ, Zimmerman G (1975) Aneurysm of saphenous vein bypass graft to coronary artery. J Thorac Cardiovasc Surg 70: 358359. [Crossref]

3. Neitzel GF, Barboriak JJ, Pintar K, Qureshi I (1986) Atherosclerosis in aortocoronary bypass grafts. Morphologic study and risk factor analysis 6 to 12 years after surgery. Arteriosclerosis 6: 594-600. [Crossref]

4. Dieter RS, Patel AK, Yandow D, Pacanowski JP Jr, Bhattacharya A, et al. (2003) Conservative vs. invasive treatment of aortocoronary saphenous vein graft aneurysms: treatment algorithm based upon a large series. Cardiovasc Surg 11: 507-513. [Crossref]

5. Baydoun A, Sabeh MK, Abdul Rahman R, Park SJ, Parikh SA (2015) Saphenous vein graft aneurysm presenting as abdominal pain. Am J Cardiol 115: 1619-1620. [Crossref]

6. Vizzi V, Bucciarelli-Ducci C, Johnson TW, Manghat NE, Baumbach A, et al. (2015) Giant saphenous vein graft aneurysm: a complex multi-disciplinary percutaneous approach. Int J Cardiol 182: 384-386. [Crossref]

7. Castañer E, Gallardo X, Rimola J, Pallardó Y, Mata JM, et al. (2006) Congenital and Acquired Pulmonary Artery Anomalies in the Adult: Radiologic Overview. Radiographics 26: 349-371. [Crossref]

8. Coselli JC, LeMaire SA (2014) Schwartz's principles of surgery. Part II, Chapter 21 Specific considerations.

9. Avrama R, Elsamalotya H, Kleshinskib JF, Williamsb JA, Assalyb R (2008) Coronary artery bypass graft pseudoaneurysm: Report of three cases. Eur J Radiol 67: e63-e67.

10. Puri R, Dundon BK, Leong DP, Worthley SG, Worthley MI (2009) Giant Saphenous Vein Graft Pseudoaneurysm Rupture Presenting with Cardiac Tamponade. Heart Lung Circ 18: 52-61. [Crossref]

11. Smith P, Qureshi S, Yacoub MH (1983) Dehiscence of infected aortocoronary vein graft suture lines. Cause of late pseudoaneurysm of ascending aorta. Br Heart J 50: 193-195. [Crossref]

12. Wyatt DA, Gay SB, Gimple LW, Spotnitz WD (1993) Successful preoperative diagnosis and treatment of a saphenous vein coronary artery bypass graft aneurysm. Chest 104: 283-284. [Crossref]

13. Almanaseer Y, Rosman HS, Kazmouz G, Giraldo AA, Martin J (2005) Severe dilatation of saphenous vein grafts: a late complication of coronary surgery in which the diagnosis is suggested by chest X-ray. Cardiology 104: 150-155. [Crossref]

14. Topaz O (2006) Giant aneurysms of saphenous vein grafts: management dilemmas and treatment options. Catheter Cardiovasc Interv 67: 617-618. [Crossref]

15. Wester DJ1, Martinez HO, Camp A (1993) Aneurysm of a saphenous vein graft manifested as a mediastinal mass on chest radiographs. AJR Am J Roentgenol 161: 951-952. [Crossref]

16. Mohara J, Konishi H, Kato M, Misawa Y, Kamisawa O, et al. (1998) Saphenous vein graft pseudoaneurysm rupture after coronary artery bypass grafting. Ann Thorac Surg 65: 831-832. [Crossref]

17. Williams ML, Rampersaud E, Wolfe WG (2004) A man with saphenous vein graft aneurysms after bypass surgery. Ann Thorac Surg 77: 1815-1817. [Crossref]

18. Kavanagh EC, Hargaden G, Flanagan F, Murray JG (2004) CT of a ruptured vein graft pseudoaneurysm: an unusual cause of superior vena cava obstruction. $A J R A m J$ Roentgenol 183: 1239-1240. 
19. Ropers D, Kuhlmann A, Weyand M, Faller G, Flachskampf FA, et al. (2004) Giant True Aneurysms of Saphenous Vein Bypass Grafts A Rare Cause of Tumor-Like Mediastinal Masses. Circulation 109: e322-e323

20. Chiappini B, Poncelet A, Noirhomme P, Verhelst R, Rubay J, et al. (2006) Giant aneurysm of aortocoronary saphenous vein graft compressing the left pulmonary artery. J Card Surg 21: 425-427. [Crossref]

21. Mitchell MB, Campbell DN (2000) Pulmonary Artery Compression by a Gian Aortocoronary Vein Graft Aneurysm. Ann Thorac Surg 69: 948-949. [Crossref]

22. Austin D, Asopa S, Owens AW, Hall JA (2011) Compression of Main Pulmonary Artery by Giant Saphenous Vein Graft Aneurysm. Ann Thorac Surg 92: 742.
23. Jeon DS, Miyamoto T, Fontana G, Friedman A, Siegel RJ (2002) Pulmonary artery compression by asaphenous vein graft aneurysm and contras techocardiography using an agitated mixture of ten percent air, ten percent blood, and ten percent saline. $J \mathrm{Am}$ Soc Echocardiogr 15: 1529-1532. [Crossref]

24. Elkington AG, Hall RJC, Mohiaddin RH (2002) Saphenous vein graft aneurysm presenting as an anterior mediastinal mass. Heart 88: 86. [Crossref]

25. Dieter RS, Patel AK, Yandow D, Pacanowski JP Jr, Bhattacharya A, et al. (2003) Conservative vs. invasive treatment of aortocoronary saphenous vein graft aneurysms: Treatment algorithm based upon a large series. Cardiovasc Surg 11: 507-513. [Crossref]

Copyright: (C2017 Jaara E. This is an open-access article distributed under the terms of the Creative Commons Attribution License, which permits unrestricted use, distribution, and reproduction in any medium, provided the original author and source are credited. 